\title{
Erratum to: Expression Analysis of Anthocyanin Biosynthetic Genes in Different Colored Sweet Cherries (Prunus avium L.) During Fruit Development
}

\author{
Yun Liu $\cdot$ Xinjie Shen $\cdot$ Kai Zhao $\cdot$ Yue Ben $\cdot$
}

Xinwei Guo $\cdot$ Xiaoming Zhang $\cdot$ Tianhong Li

Published online: 30 August 2013

(c) Springer Science+Business Media New York 2013

\section{Erratum to: J Plant Growth Regul}

DOI 10.1007/s00344-013-9355-3

The article note to designate the order of contributing authors was not submitted and published.

The missing article note is given below:

"Yun Liu and Xinjie Shen are the co-first authors of the paper".

The online version of the original article can be found under doi:10.1007/s00344-013-9355-3.

Y. Liu $\cdot$ X. Shen $\cdot$ K. Zhao $\cdot$ X. Guo $\cdot$ T. Li $(\bowtie)$

Department of Pomology, Key Laboratory of Stress Physiology and Molecular Biology for Tree Fruits of Beijing, College of

Agronomy and Biotechnology, China Agricultural University, Beijing 100193, People's Republic of China

e-mail: lith@cau.edu.cn

Y. Liu

e-mail: whbeuty@163.com

Y. Ben

Beijing No. 101 Middle School, Haidian, Beijing 100091, People's Republic of China

X. Zhang

Institution of Forestry and Pomology, Beijing Academy of Agriculture and Forestry Science, Beijing 100093, People's Republic of China

T. $\mathrm{Li}$

No. 2, Yuanmingyuan West Road, Haidian, Beijing 100194,

People's Republic of China 